\section{Csk quenches the fire}

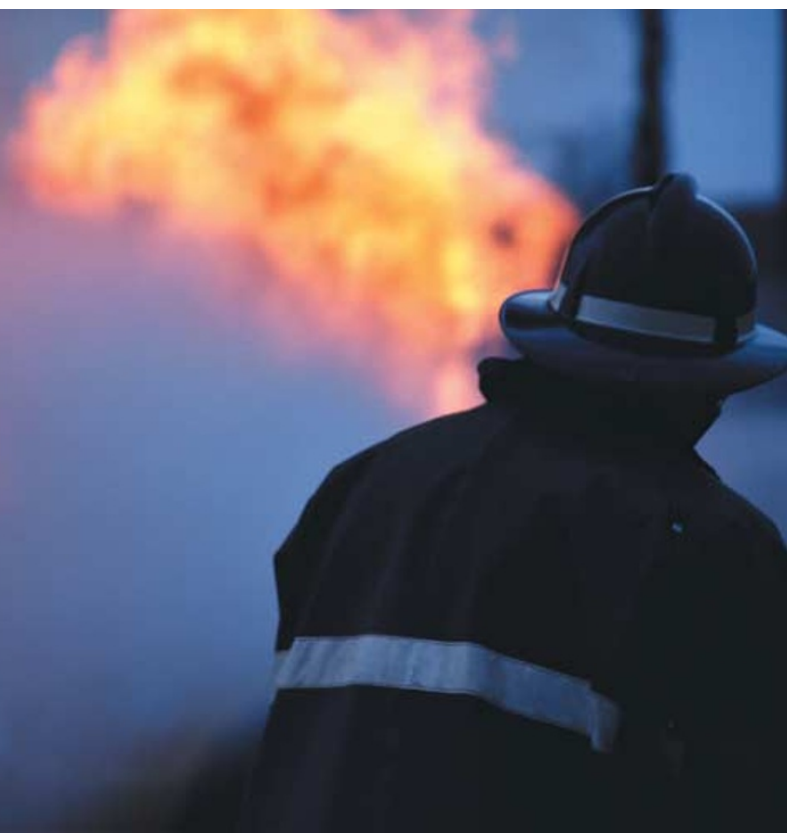

The ligation of integrins on phagocytes triggers a signalling cascade that involves the Src family of tyrosine kinases and is a crucial step in the initiation of inflammatory responses. However, little was known about the regulation of Src-family kinases during inflammation, until a study published recently in Immunity identified carboxy-terminal Src kinase (Csk) as a key negative regulator of these kinases in inflammatory cells.

Csk-deficient mice die in utero. So, to study the role of Csk during acute inflammation, Thomas et al. used a conditional mutagenesis strategy to eliminate Csk in granulocytes (Csk-GEcre). Acute inflammation of the skin and lungs was observed in Csk-GEcre mice housed in conventional facilities, but this was ameliorated by treatment with antibiotics or rederivation of the mice in a specific-pathogen-free environment. Further evidence of a role for Csk in inflammation was indicated by the fact that Csk-GEcre mice showed an enhanced susceptibility to normally sub-lethal doses of lipopolysaccharide, and this was associated with increased serum levels of tumour-necrosis factor.

The hyperresponsiveness of the Csk-GEcre mice correlated with an increased responsiveness of Cskdeficient bone-marrow granulocytes in vitro; culturing in the absence of stimuli induced spontaneous degranulation and upregulation of expression of $\beta_{2}$ and $\beta_{3}$ integrins, as did pharmacological stimulation with PMA and crosslinking of cell-surface integrins. The effect of this increased integrin expression on Csk-deficient granulocyte function was markedly enhanced adhesiveness and decreased chemotaxis, resulting from enhanced actin polymerization and remodelling of the cytoskeleton. Together, these data indicate a negative role for Csk in granulocyte activation.

This increased responsiveness of Csk-deficient bone-marrow granulocytes in vitro was repressed by the inhibitor of Src-family kinases PP2, indicating that hyperactivity in the absence of Csk is mediated, at least in part, by the initiation of

\section{T-CELL ACTIVATION}

\section{How to wake a sleeping lymphocyte}

\author{
According to new research published in \\ Science, downregulation of the Fox \\ (forkhead)-family transcription factor Foxj1 \\ in the lymphoid system can make naive \\ T helper $\left(T_{H}\right)$ cells more responsive \\ to the activating signals that wake them \\ from their slumber. \\ These investigations arose from the initial \\ observation that Foxj1 is downregulated \\ in lymphocytes from mice that are prone \\ to systemic lupus erythematosus. Having \\ shown that Foxj1 is highly expressed by \\ wild-type naive $\mathrm{T}$ cells but is rapidly \\ downregulated when the cells are \\ stimulated, the authors set out to examine \\ the role of this factor in the regulation of \\ immune responses. As most mice with Foxj1 \\ mutations die in utero or soon after birth, \\ they transplanted Foxj $1^{-1-}$ fetal livers into \\ $\mathrm{Rag}^{-1-}$ mice to generate chimeric animals \\ with a Foxj1 $1^{-/}$lymphoid system. Initial \\ analyses showed that these mice had \\ systemic autoimmune inflammation with \\ lymphocytic infiltrates of various organs.
}

T-cell activation is in part controlled by the transcription factor nuclear factor- $\mathrm{KB}$ (NF-KB), and this study went on to show that Foxj1 is an upstream inhibitor of spontaneous and inducible NF- $\kappa \mathrm{B}$ activity in cell lines. Furthermore, Foxj1 $1^{-1-} \mathrm{T}_{\mathrm{H}}$ cells had increased spontaneous NF- $\kappa B$ activity, and blocking NF- $\kappa B$ prevented the hyperresponsiveness of these cells. The inhibitory effect of Foxj1 was probably the result of upregulation of $I \kappa B \beta$, which, as part of the inhibitor of NF- $\kappa \mathrm{B}(\mathrm{I} \kappa \mathrm{B})$ complex, prevents the translocation of NF- $\kappa B$ to the nucleus.

It seems that Foxj1 maintains naive $\mathrm{CD}^{+}$ $\mathrm{T}_{\mathrm{H}}$ cells in a quiescent state by inhibiting NF- $\kappa B$ activity, thereby preventing inappropriate and possibly self-reactive inflammatory responses. This study adds to the known role of other Fox-family members in the immune system, such as Foxp3 in regulatory T cells, Foxo proteins in lymphocyte proliferation and apoptosis, and Foxn 1 in thymic epithelial-cell development.

\section{Kirsty Minton}

(i) References and links ORIGINAL RESEARCH PAPER Lin, L. et al. Modulation of Th1 activation and inflammation by the NF-KB repressor Foxj1. Science 303, 1017-1020 (2004) FURTHER READING Li, Q. \& Verma, I. M. NF-kB regulation in the immune system. Nature Rev. Immunol. 2, 725-735 (2002) 
Src-family kinase signalling cascades. Confirmation of this was provided by the observation that the Src-family kinase substrates cortactin and paxillin were hyperphosphorylated in Cskdeficient bone-marrow granulocytes cultured in vitro. In addition, hyperphosphorylation of the tyrosine kinase Syk - a mediator of the proximal stages of integrin-signalling pathways — was also detected.

These data indicate that Csk negatively regulates granulocyte activation, modulating cellular adhesion and preventing inappropriate granulocyte recruitment. As the phenotype of Csk-GEcre mice is similar to several inflammatory skin conditions of unknown aetiology in humans, the authors suggest that impaired negative regulation of granulocyte activation might have a role in the induction of these inflammatory conditions.

Karen Honey

(Q) References and links ORIGINAL RESEARCH PAPER Thomas, R. M. et al. C-terminal Src kinase controls acute inflammation and granulocyte adhesion. Immunity 20, 181-191 (2004)

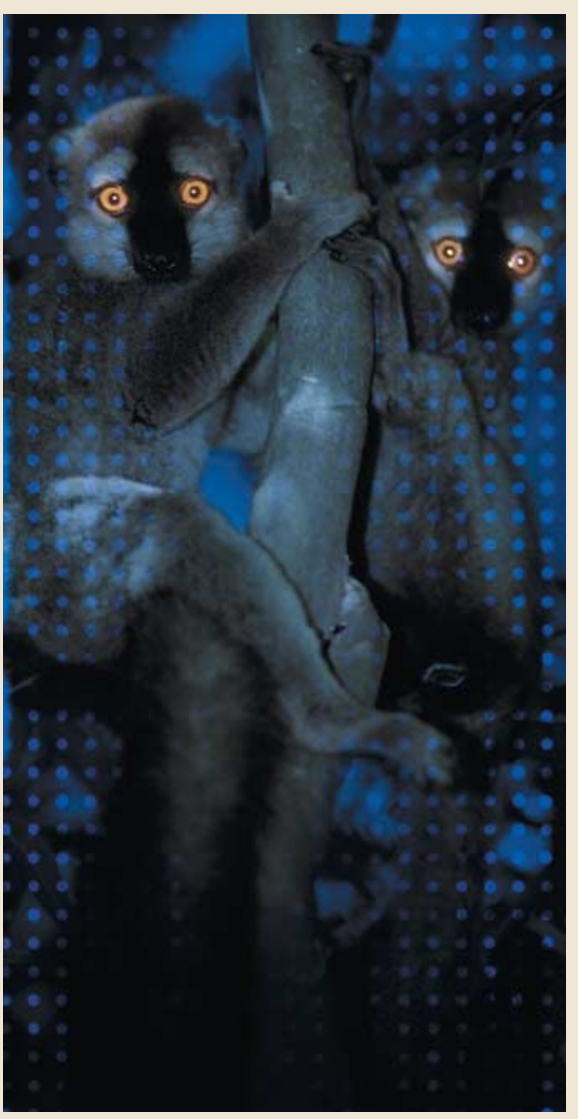

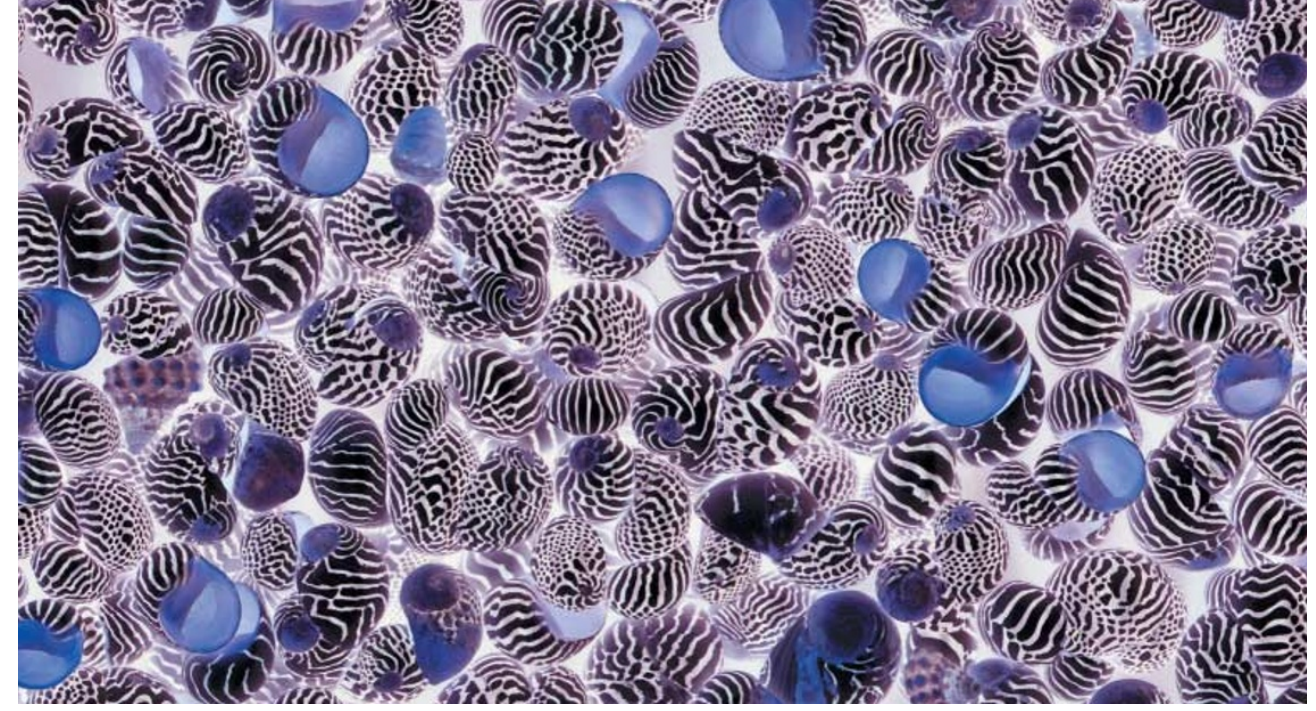

INNATE IMMUNITY

\section{TLR ligands from the natural world}

Toll-like receptor 7 (TLR7) and TLR8 are known to be activated by ribonucleoside analogues; however, the identity of their natural ligands had remained undefined until two reports in Science characterized single-stranded (ss) RNA as this ligand.

The innate immune response to influenza virus is exemplified by the production of type 1 interferons (IFN- $\alpha / \beta)$ ). Although conventional dendritic cells (DCs) can produce high levels of IFN- $\alpha$ in response to double-stranded (ds) influenza RNA - an intermediate of viral genome replication - this response is suppressed during infection by a virus-encoded dsRNA-sequestering protein. By contrast, the production of IFN- $\alpha$ by plasmacytoid DCs (pDCs) is resistant to suppression, indicating that it can occur by a dsRNA-independent mechanism.

Diebold et al. set out to identify this influenza-virus recognition pathway in pDCs. Initial analysis showing that IFN- $\alpha$ production by wild-type pDCs was abolished in the presence of chloroquine, combined with the observation that pDCs lacking the TLR-adaptor molecule MYD88 did not produce IFN- $\alpha$ in response to influenza virus, indicated that a TLR molecule known to have endosomal localization was likely to be pivotal in viral recognition. Although TLR3 and TLR9 are known to reside in the endosomal compartment, $\mathrm{pDCs}$ derived from mice deficient in these receptors made normal IFN- $\alpha$ responses to influenza virus. By contrast, this response was abolished in the absence of TLR7.

As TLR7 signalling is known to be induced by ribonucleoside analogues, the authors assessed the ability of purified influenza virus genomic ssRNA to induce IFN- $\alpha$ production by pDCs, and showed that viral ssRNA was a ligand for TLR7. Interestingly, ssRNA of non-viral origin, such as poly-uridine (polyU) - but not other nucleic-acid oligomers - and in vitrosynthesized RNA encoding green fluorescent protein, induced a TLR7-dependent IFN- $\alpha$ response by pDCs, indicating that ssRNA is the natural ligand for TLR7.

A similar observation was made independently by Heil et al., whose studies were initiated to investigate whether ssRNA might act as a ligand for the TLR9 subfamily of TLRs: TLR7, TLR8 and TLR9. The authors first observed that only nucleosides composed of guanine $(G)$ and $U$ induced human peripheralblood mononuclear cells (PBMCs) to secrete inflammatory cytokines. However, if the RNA a synthetic RNA containing the GU-rich sequence in the U5 region of HIV-1 - was complexed in such a way as to facilitate uptake by DCs,

IFN- $\alpha$ was also produced. This IFN- $\alpha$ response was restricted to the pDC population. Similarly, GU-rich ssRNA derived from HIV-1 selectively induced mouse $\mathrm{pDCs}$ to produce IFN- $\alpha$.

The involvement of TLRs in this process was confirmed using MYD88-deficient DCs, which failed to respond to ssRNA stimulation. Additionally, analysis of DCs derived from TLR3-, TLR9-, TLR8- and TLR7-deficient mice indicated that, in mice, only TLR7 recognizes GU-rich ssRNA. By contrast, in an in vitro culture system, only cells expressing human TLR8, and not TLR7, were activated in response to ssRNA, indicating that there is species-specific recognition of GU-rich ssRNA.

These studies identify ssRNA as the natural ligand for mouse TLR7 and human TLR8, and the authors of both papers suggest that endosomal delivery of ssRNA could provide a novel adjuvant for vaccination and immunotherapy.

Karen Honey

\section{6) References and links}

ORIGINAL RESEARCH PAPERS Diebold, S. S. et al. Innate antiviral responses by means of TLR7-mediated recognition of single-stranded RNA. Science 303, 1529-1531 (2004) | Heil, F. et al. Species-specific recognition of single-stranded RNA via Toll-like receptors 7 and 8 . Science 303, 1526-1529 (2004) 